\title{
An Overview of Cancer Thermal Therapy Technology Based on Different Types of Antenna Exposure
}

\author{
Kasumawati Bt Lias, Norlida Buniyamin \\ Faculty of Electrical Engineering \\ Universiti Teknologi MARA, \\ Shah Alam, Selangor \\ danza252@gmail.com,nbuniyamin@salam.uitm.edu.my
}

\author{
Mohd Zulkarnaen B Ahmad Narihan \\ Faculty of Medical and Health Science, \\ Universiti Malaysia Sarawak \\ anmzulkarnaen@fmhs.unimas.my
}

\begin{abstract}
Cancer thermal therapy is a treatment that uses heat generated using Electromagnetic (EM) fields. This treatment, significantly increase the effectiveness of radiotherapy or chemotherapy treatment towards cancerous tissue or cell. The main goal of this paper is to provide an overview of previous and present technology on cancer thermal therapy which is based on the application of various types of antenna exposure towards cancerous tissue or cell.
\end{abstract}

The paper also proposes a new antenna design that increases the focusing capability and significantly reduces the skin burn effect through the introduction of metamaterial. In addition, the establishment of an antenna with the integration of Electromagnetic Band Gap (EBG) will facilitate depth penetration with selective frequencies. The new antenna design is be expected to significantly improve the specific absorption rate (SAR) distribution.

Keywords- thermal therapy; hyperthermia; antenna; cancerous cell; electromagnetic fields

\section{INTRODUCTION}

Cancer is the growth and spread of uncontrolled abnormal cells that forms malignant tumours and invades nearby parts of the body. In 2008, World Health Organization (WHO) estimated 12.66 million people were diagnosed with cancer across the world and 7.56 million people died from the disease [1]. Meanwhile, in Malaysia, 18,219 cancer cases were diagnosed among Malaysians in Peninsular Malaysia in the year 2007 and registered in the National Cancer Registry. It comprises of 8,123 and 10,096 of males and females, respectively [2].

Nowadays, proliferate of cancerous cells can be destroyed or killed by radiotherapy, chemotherapy, hormone therapy and surgery. Recently, thermal therapy using EM fields has been introduced as an alternative therapy to manage various types of cancers.

\section{CANCER THERMAL THERAPY}

Cancer thermal therapy using EM is part of the application in bioelectromagnetics where EM fields are applied towards human biological tissue. Cancer thermal therapy basically utilizes thermal ablation such as microwave (MW), radio frequency (RF) and high intensity focused ultrasound. In addition, thermal therapy also associates to hyperthermia. Thermal ablation and hyperthermia are able to destruct cancerous tissue or cells. Thermal ablation and hyperthermia elevate temperature to greater than $50^{\circ} \mathrm{C}$ and into a range of $41-45^{\circ} \mathrm{C}$, respectively [3]. This paper emphasized on thermal therapy particularly the use of hyperthermia in treatment of cancer. Besides thermal ablation, hyperthermia is a promising [4] treatment for cancer. Many researchers, for example, J. Van Der Zee [5], Y. Harima [6], D. E. Thrall [7], S. Triantopoulou [8], C. Marchal [9] and Y. Hiraki [10] has shown that hyperthermia improve the effectiveness in treating cancerous tissues or cells with adjuvant treatment of radiotherapy plus hyperthermia.

In 1986, Busch [11], carried out a research in hyperthermia that indicated certain temperatures might selectively destroyed tumor tissue. Currently research in hyperthermia draws interest among researchers around the globe and the area of research in hyperthermia can be divided into antenna design, focusing techniques, microwave applicator and clinical experiments on swine or rats. Research is currently carried out through computational simulation or experimentation or combination of both. Figure 1 shows the number of hyperthermia research that has been conducted either through simulation or experimentation/clinical.

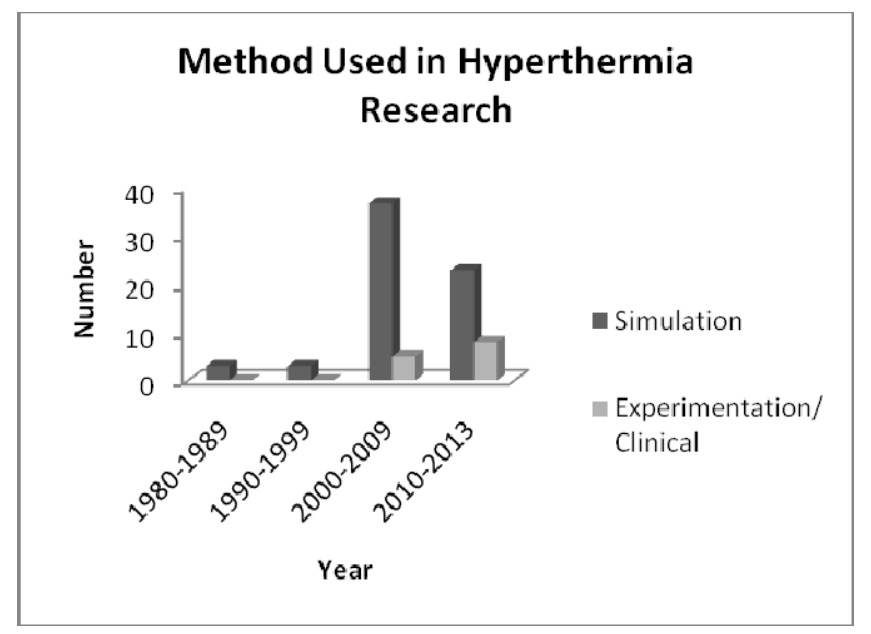

Figure 1. Number of Hyperthermia Research conducted. 